\title{
EMI Signal Classification Based on Improved HHT and CNN
}

\author{
Hongyi $\mathrm{Li}^{1}$, Di Zhao ${ }^{1,+}$ and Kejia $\mathrm{Hu}^{1}$ \\ ${ }^{1}$ LMIB, School of Mathematical Sciences, Beihang University, Beijing 100191, China
}

\begin{abstract}
This paper proposes a new single channel EMI signal classification method. In this method, we propose to use an improved HHT algorithm, BEMD algorithm and HT algorithm, to convert nonlinear and non-steady-state single-channel electromagnetic interference signals into time-frequency domain matrices, which solves the problem of traditional EMD algorithms. After that, we use the time-frequency domain matrix as the input of the CNN to classify the EMI signals. By the simulation signal experiment and EEG signal experiment verification, the proposed algorithm has a good classification effect.
\end{abstract}

Keywords: Signal classification, HHT, EMD, EMI.

\section{Introduction}

With the development of technology, the number of electronic devices in various fields is increasing, toward high frequency, wide frequency band. At the same time, the electromagnetic interference problem caused by a large number of electronic devices has gradually become one of the non-negligible problems in aerospace field [1], medical field [2], audio system[3]. Generally speaking, there are many types of electromagnetic interference signals, and the separation is complicated, therefore it is necessary to classify electromagnetic interference signals.

Usually, the electromagnetic interference signal is single channel. However, the direct classification model for single-channel timing signals has a poor learning effect. Traditional signal decomposition methods, such as Fourier transform and wavelet transform, are often used as signal processing methods, but Fourier transform is a linear integral transform method that is not applicable to nonlinear signal processing. Wavelet transforms cannot handle unsteady signals. In order to solve the problem of nonlinear and unsteady signal processing like EMI, Huang proposed the empirical mode decomposition method (EMD) [4]. So far, the EMD method has been applied in several aspects. As an application of the EMD algorithm, Huang and Hilbert jointly proposed the HHT algorithm. The HHT algorithm is actually a combination of the EMD algorithm and the Hilbert transform.

However, due to the different time scales in the EMI signal, there is a mixed- mode problem in the EMD algorithm. The mixed mode problem will affect the establishment of the subsequent classification model to some extent. In order to solve the mixed-mode problem of EMD algorithm and improve the efficiency of the algorithm, Hongyi Li et al. proposed a B-spline empirical mode decomposition method [5], which not only improves the accuracy of signal separation, but also reduces the calculation consumption of EMD algorithm. As a signal classification algorithm, we propose a method based on the improved HHT algorithm (BEMD+HT)+CNN. CNN [6] s a deep learning neural network widely used in many other fields [7-9].

The main innovation is that an improved HHT algorithm is proposed and applied to EMI signal single channel to multi-channel processing. The EMD algorithm in HHT is replaced by the BEMD algorithm. The cubic B-spline interpolation method is used instead of the EMD original process.

The following content of this article will be expanded in the following order. In the second part we will introduce the HHT algorithm. In the third part we will introduce the improved HHT algorithm. In the fourth part we will introduce the basic principles of $\mathrm{CNN}$ and the network structure. The last part is the conclusion.

\footnotetext{
+ Corresponding author.

E-mail address: zdhyl2010@163.com.
} 


\section{HHT Algorithm}

\subsection{Empirical Mode Decomposition}

The main process of the EMD algorithm is as follows:

1) The maxima and minima points of the signal $\mathrm{s}(\mathrm{t})$ are respectively obtained. Take the mean of the two envelopes to get a mean curve.

2) Determine if the mean curve is IMF. It must satisfy two conditions: First, the mean of the mean curve must be equal to 0 . Second, the number of extreme points of the original signal and the intersection of the original signal and the $\mathrm{x}$-axis are less than or equal to 1 .

3) If the mean curve is IMF, then the IMF is stripped from the original signal

$$
s_{i+1}(t)=s_{i}(t)-I M F_{i}(t)
$$

If the mean curve is not an IMF, repeat the first three steps with the IMF as a new signal.

4) Judge whether there is a missing point or a minimum point in $\mathrm{s}(\mathrm{t})$. If not missing, skip to the beginning step, otherwise end the program and get

$$
s_{0}(t)=\sum_{i=1}^{k} I M F_{i}(t)+r_{k}(i)
$$

\subsection{Hilbert transform and Hilbert spectrum analysis}

The Hilbert transform actually dose a convolution of $x(t)$ with $1 / \pi t$, and makes Hilbert transform of $x(t)$ into $H(x(t))$

$$
H(x(t))=\frac{1}{\pi} \int_{-\infty}^{\infty} \frac{x(\tau)}{t-\tau} d \tau
$$

Use HT to construct analytic $z(t)$ for $s(t)$, define as follows:

$$
z(t)=s(t)+i H(s(t))=a(t) e^{i \theta(t)}
$$

Among them, $i=\sqrt{-1}, a(t)=\left(s^{2}(t)+H^{2}(s(t))\right)^{2}$ and $\theta(t)=\tan ^{-1} \frac{H(s(t))}{s(t)} \cdot a(t)$ and $\theta(t)$ are instantaneous amplitude and instantaneous phase functions of $s(t)$, The instantaneous frequency of the analytical signal can be found from $\theta(t)$ :

$$
\omega(t)=\frac{d \theta(t)}{d t}
$$

The EMD generates each $I M F_{i}$ as input $s(t)$ to obtain the instantaneous frequency function $\omega_{i}(t)$ and the instantaneous amplitude function $a_{i}(t)$ of each $\mathrm{IMF}_{\mathrm{i}}$, and calculate the time-frequency domain matrix $H_{i}(\omega, t)$ of each $I M F_{i}$. The third dimension represents the instantaneous amplitude, then the time-frequency domain function of the entire signal is $H(w, t)$

$$
H(w, t)=\sum_{i=1}^{N} H_{i}(\omega, t)
$$

\section{Improved HHT Algorithm}

The improved HHT algorithm modifies the EMD part of the HHT algorithm and replaces the EMD part with the BEMD algorithm. BEMD is an improved EMD algorithm. The efficiency and adaptability of the BEMD algorithm are higher than the EMD algorithm. The following mainly introduces the BEMD algorithm, and Improved HHT algorithm for the processing of EMI signals.

\subsection{BEMD Algorithm}

The main improvement of the BEMD algorithm is in the process of generating the mean curve. The algorithm flow is as follows:

1) Find all the extreme points $s(t)$, including all the minimum and maximum points, and then calculate the midpoint of all the two adjacent points.

2) Interpolate all midpoints using cubic B-spline interpolation to obtain a mean curve.

3 ) It is judged whether the mean value of the mean curve is equal to 0 (the difference from 0 is less than 0.1 , and the number of extreme points of the original signal and whether the intersection of the original signal and the $\mathrm{x}$-axis is less than or equal to 1 . 
4) If the above two conditions are not met, repeat the above two steps as the new signal curve. If the above two conditions are met, the mean curve is IMF and the IMF is stripped from $s(t)$. Use $r_{i}(t)$ as the new signal and repeat the above process until $r(t)$ leaves only a certain number of extreme points.

$$
r_{i}(t)=s(t)-I M F_{i}(t)
$$

In terms of adaptability, the BEMD algorithm also has better adaptability than the EMD algorithm. The following figure compares the EMD signals of the EMD and BEMD algorithms. It can be seen from the figure that the first three IMFs separated by the BEMD algorithm solve the mixed mode problem.
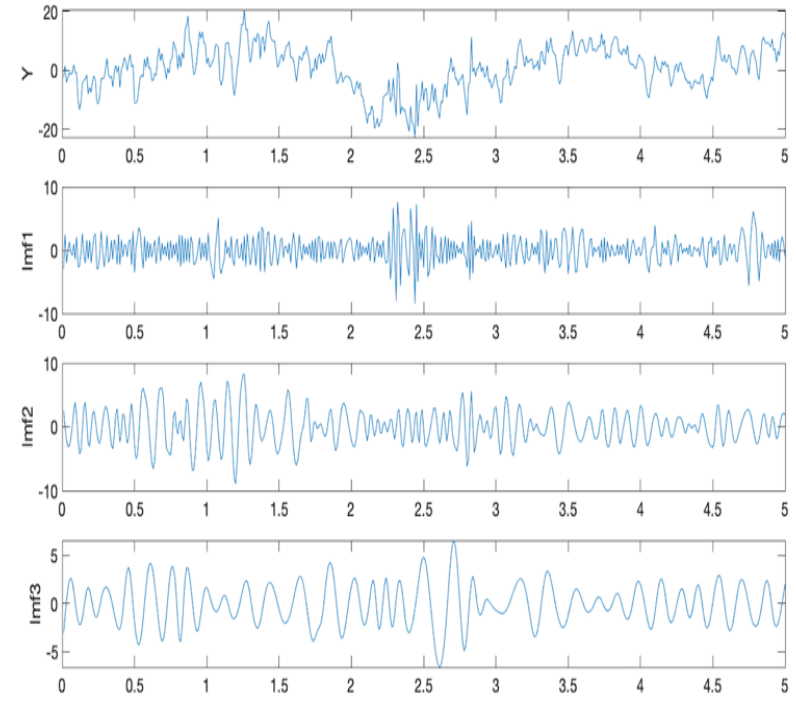

(a)
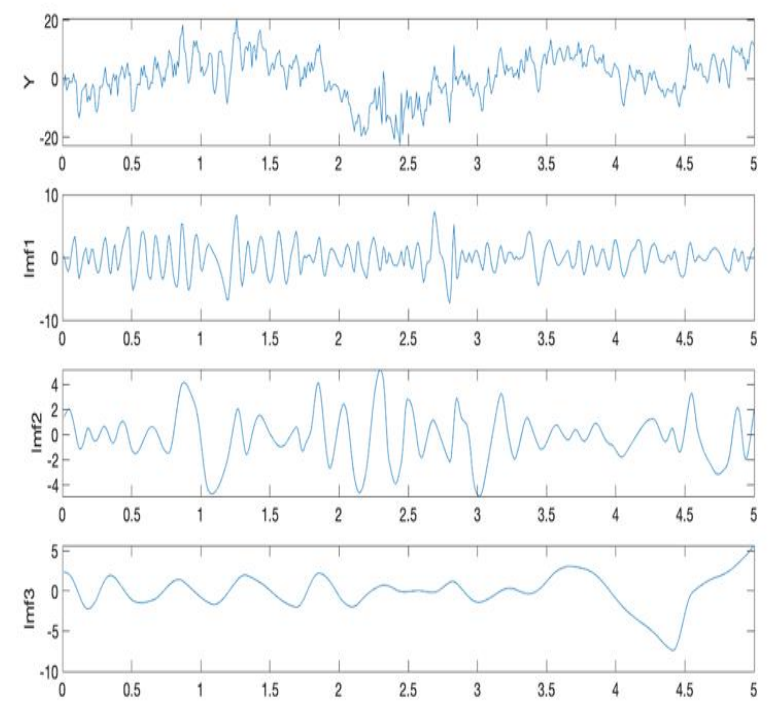

(b)

Fig. 1: Sample of comparison between BEMD and EMD (a) BEMD, (b) EMD.

\subsection{Processing of EMI Signals}

Through the BEMD algorithm, we obtain IMFs for each single-channel signal, and obtain the HT transform of HHT algorithm. The time-frequency domain matrix of the entire signal, as an input to the CNN.

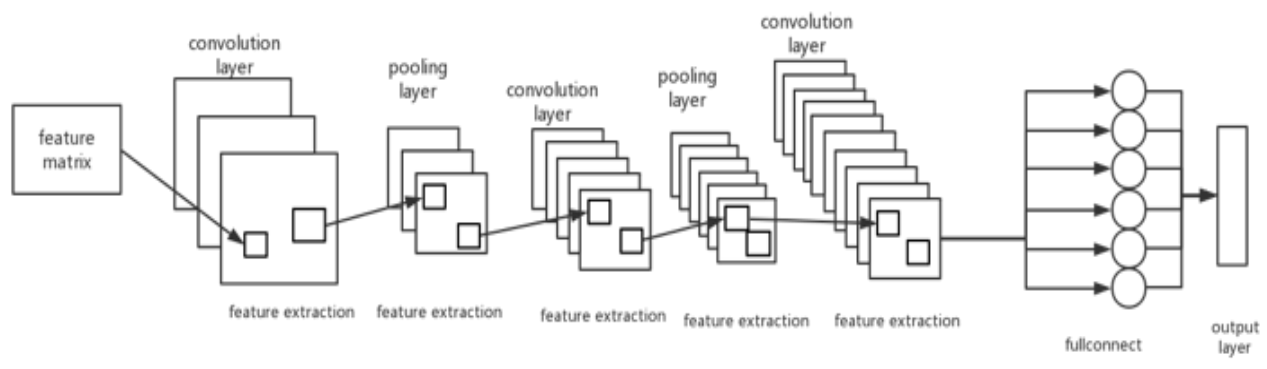

Fig. 2: CNN structure used in the article.

\section{Convolutional Neural Network}

The convolutional neural network mainly consists of two parts, one part is the feature extraction layer, including the convolution layer, the pool layer and other neural layers. First, unlike traditional fullyconnected neural networks, convolutional networks are sparsely interacting. By convolution, a time axis consisting of the moments at which different features appear in the input can be obtained. In the pooling layer, we use the pooling function to take the adjacent output statistical feature of a certain location as the output of the location. Since the pooling integrates information of several adjacent data, it also reduces the input scale and extracts the data characteristics. The fully connected layer of the convolutional neural network trains and reduces the extracted features of the CNN feature extraction layer, and finally obtains the output result. 
In summary, the convolutional layer and the pooled layer can extract effective feature information from the high-dimensional feature matrix, while the fully-connected layer can map the high-dimensional matrix features to the low-dimensional space, and the mapped feature vector is saved. A large amount of local information of high-dimensional matrix features has a good performance in timing problems. The CNN network structure used in this paper is Fig. 2.

\section{Experimental Design and Result}

\subsection{EMI Simulation Signal Experiment}

In order to test the effect of the improved HHT+CNN model, we used a simulated signal with Gaussian white noise for experimentation. There are 200 sets of experimental data in the experiment. For each $s(t)$, a AWGN $n(t)$ with SNR (signal-to-noise) of $10 \mathrm{~dB}$ is added to obtain the signal $d(t)=s(t)+n(t)$.

All simulated signals have a frequency of $100 \mathrm{~Hz}$. The basic functions of the first set of simulated signals for the experimental data are $y_{1}(t)=3 \cos \left(5 \pi t+\frac{\pi}{2}\right), y_{2}(t)=2 \cos (4 \pi t), y_{3}(t)=\sin (2 \pi t)$ and $s_{1}(t)=y_{1}(t)+y_{2}(t)+y_{3}(t)$. The basic functions of the second set of simulated signals for the experimental data are $s_{2}(t)=y_{1}(t)+y_{2}(t)$.

The basic functions of the third set of simulated signals for the experimental data are

$$
s_{3}(t)=\cos (2 \pi t)+\cos (20 \pi t+2 \sin (2 t))+\cos (500 \pi t+2 \sin (2 t))
$$

The basic functions of the fourth set of simulated signals for the experimental data are

$$
s_{4}(t)=(1+\cos (\pi t))-\cos (2 \pi t)+\cos (500 \pi t+2 \sin (2 t))+\cos (500 \pi t+2 \sin (2 t))
$$

The following figure shows the signal diagram of four sets of data.
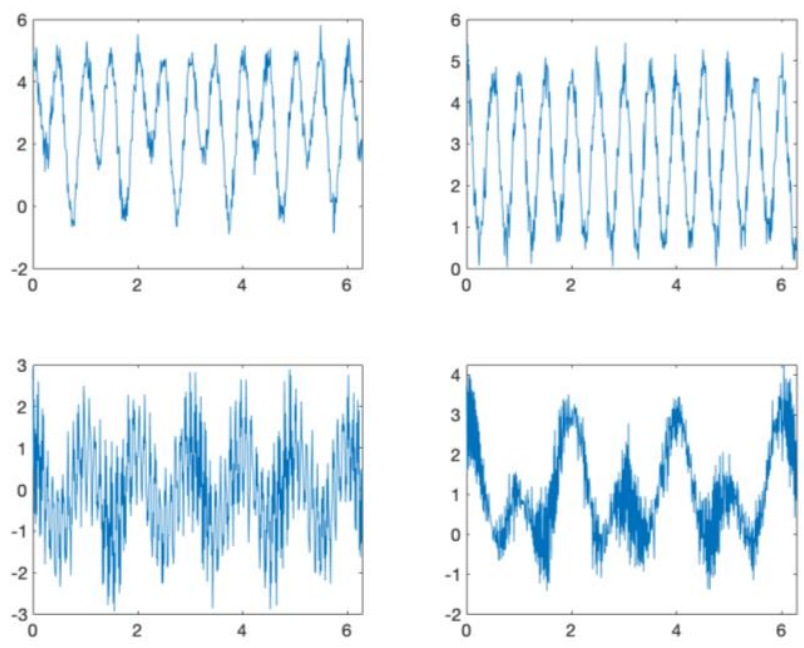

Fig. 3: Sample of simulation signal.

In order to compare with the algorithm of BEMD $+\mathrm{HT}+\mathrm{CNN}$ proposed in this paper, the experiments used logit boost [10], HHT+CNN [8], CNN [9], DNN [11] to test the data. All model experimental results were obtained by 10 cross validations. The simulation results are in Table 1.

Table 1: EEG signal experiment result.

\begin{tabular}{|c|c|c|c|c|c|}
\hline Algorithm & Logitboost[10] & HHT+CNN[8] & CNN [9] & DNN [11] & $\begin{array}{c}\text { BEMD+HT+C } \\
\text { NN (proposed) }\end{array}$ \\
\hline Accuracy & $86.17 \%$ & $92.13 \%$ & $90.36 \%$ & $87.53 \%$ & $\mathbf{9 4 . 0 7 \%}$ \\
\hline
\end{tabular}

\subsection{EEG Data Set Experiment}

In order to test the application of the proposed algorithm to the separation of electromagnetic interference signals, we selected a set of public EEG data sets as test samples. The EEG test set [5] was generated by a Biosemi Active 2 system with a frequency of $2048 \mathrm{~Hz}$. The data set is a total of two classification sets. The non-P300 signal can be used as the electromagnetic interference signal to simulate the 
electromagnetic interference signal. We connect several signals of different channels as a single channel to calculate the performance of the algorithm proposed. The following figure shows the signal acquisition map of the first eight channels of the p300 signal.
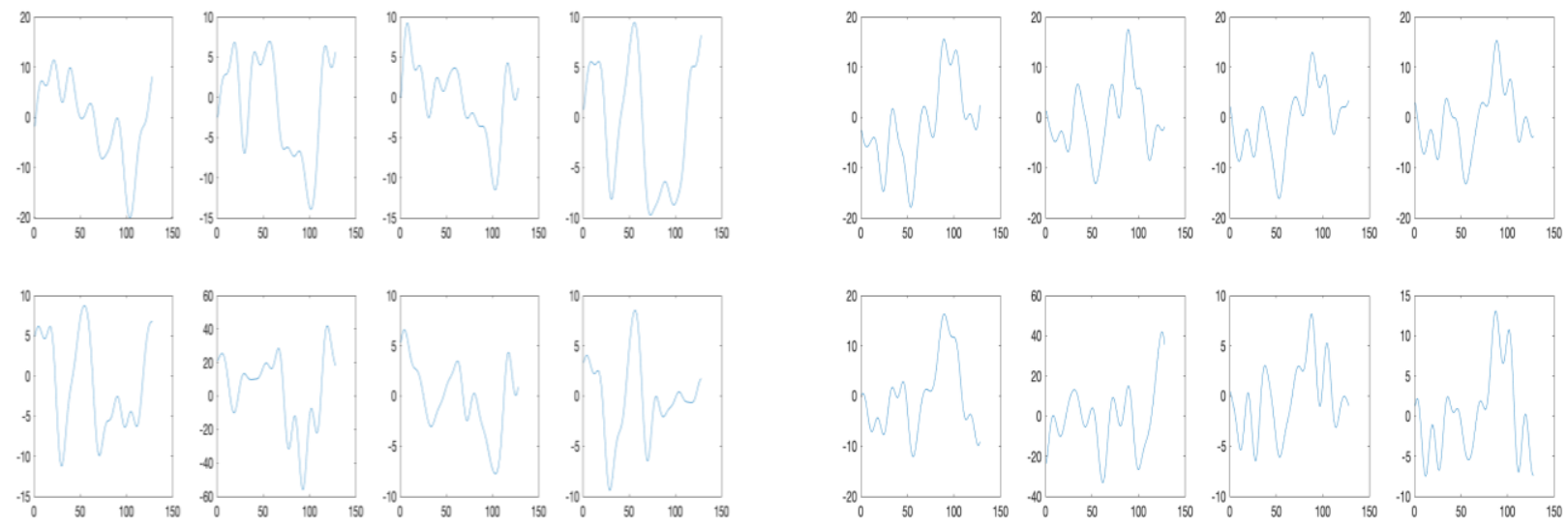

(a)

(b)

Fig. 4: Sample of main signal and electromagnetic interference signal

(a) main signal, (b) electromagnetic interference signal.

The experimental platform of this experiment is MATLAB2017b. The experiment uses a 9:1 proportional segmentation experimental data set to evaluate the applicability of the improved $\mathrm{HHT}+\mathrm{CNN}$ model. The same data set will be used for BEMD+HT+CNN, HHT+CNN, CNN, DNN. Wait for the model to test the data. All model experimental results were obtained by 10 cross validations. The simulation results are in Table 2.

Table 2: EEG signal experiment result.

\begin{tabular}{|c|c|c|c|c|c|}
\hline Algorithm & Logitboost [10] & HHT+CNN [8] & CNN [9] & DNN [11] & $\begin{array}{c}\text { BEMD+HT+C } \\
\text { NN (proposed) }\end{array}$ \\
\hline Accuracy & $86.17 \%$ & $92.13 \%$ & $90.36 \%$ & $87.53 \%$ & $\mathbf{9 4 . 0 7 \%}$ \\
\hline
\end{tabular}

\section{Conclusion}

This paper proposes to use the improved HHT+CNN algorithm to classify EMI signals. In the improved HHT part, the BEMD algorithm is used to replace the EMD algorithm, which solves the mixed mode problem in the EMD algorithm to some extent, and thus obtains better EMI signals. Time-frequency domain matrix. The feature matrix is used as the input of CNN to classify the EMI signal. The simulation signal experiment and EEG signal experiment are compared with several existing algorithms to obtain better classification accuracy.

\section{Acknowledgements}

This work was supported by the National Natural and Science Foundation of China (Grant No. 61771001).

\section{References}

[1] J.I. Saeks. ELECTROMAGNETIC COMPATIBILITY CONSIDERATIONS FOR AEROSPACE POWER SYSTEMS. Proc. Intersoc. Energy Convers. Eng. Conf., 1985, pp. 270-274.

[2] F. Censi, G. Calcagnini, E. Mattei, M. Triventi, P. Bartolini. RFID in healthcare environment: Electromagnetic compatibility regulatory issues. in 2010 Annual International Conference of the IEEE Engineering in Medicine and Biology Society, EMBC 10 (IEEE Computer Society), 2010, pp. 352-355.

[3] P. Giddings. Introduction to electromagnetic compatibility (EMC) and electromagnetic interference (EMI) for audio system designers. AES J. Audio Eng. Soc., 1989, 37, (7-8): 570-585. 
[4] N. E. Huang, Z. Shen, S. R. Long, et al. The empirical mode decomposition and the Hubert spectrum for nonlinear and non-stationary time series analysis. Proc. R. Soc. A Math. Phys. Eng. Sci. 1998.

[5] H. Li, C. Wang, D. Zhao. An Improved EMD and Its Applications to Find the Basis Functions of EMI Signals. Math. Probl. Eng. 2015.

[6] A. Krizhevsky, I. Sutskever, G. E. Hinton. ImageNet classification with deep convolutional neural networks, in Advances in neural information processing systems, 2012: 1097-1105.

[7] Darmatasia, Fanany, M.I. Handwriting recognition on form document using convolutional neural network and support vector machines (CNN-SVM). 2017 5th Int. Conf. Inf. Commun. Technol. ICoIC7 2017.

[8] L. Wei, Y. Lin, J. Wang, Y. Ma. Time-frequency convolutional neural network for automatic sleep stage classification based on single-channel EEG. Proc. - Int. Conf. Tools with Artif. Intell. ICTAI, 2018: 88-95.

[9] C. Niederhoefer, F. Gollas, R. Tetzlaff. EEG analysis by multi-layer cellular nonlinear networks (CNN). IEEE 2006 Biomed. Circuits Syst. Conf. Healthc. Technol. BioCAS 2006: 25-28.

[10] U. Hoffmann, G. Garcia, J.-M. Vesin, K. Diserenst, T. Ebrahimi. A boosting approach to P300 detection with application to brain-computer interfaces. 2nd Int. IEEE EMBS Conf. Neural Eng., 2005: 97-100.

[11] S. Kumar, A. Sharma, K. Mamun, T. Tsunoda. A Deep Learning Approach for Motor Imagery EEG Signal Classification. Proc. - Asia-Pacific World Congr. Comput. Sci. Eng. 2016 Asia-Pacific World Congr. Eng., 2016. 